\title{
Dual-Wavelength Mode-Locked Fiber Laser With an Intracavity Silicon Waveguide
}

\author{
Xinzhu Sang, En-Kuang Tien, Nuh S. Yuksek, Feng Qian, Qi Song, and Ozdal Boyraz, Member, IEEE
}

\begin{abstract}
We demonstrate dual-wavelength short pulses lasing at 1540 and $1675 \mathrm{~nm}$ based on a silicon waveguide. The inline silicon waveguide inside a laser cavity facilitates pulse compression and laser mode-locking due to two-photon absorption (TPA) and TPA-induced free-carrier absorption at $1540 \mathrm{~nm}$. Compressed pulses provide pump for stimulated Raman scattering. Raman amplification and low threshold Raman lasing are observed based on the same silicon waveguide.
\end{abstract}

Index Terms-Nonlinear optics, optical planar wavegudie, Raman effect, silicon-on-insulator technology.

\section{INTRODUCTION}

B ECAUSE of its possible dense on-chip integration with microelectronics and significant advances in nonlinear effects, silicon photonics has become a rapidly growing field. Due to the high-index contrast between the silicon core and silica cladding, silicon waveguides allow strong optical confinement and large effective nonlinearity, which facilitates low-cost chip-scale demonstration of all-optical nonlinear functional devices at relatively low pump powers. In fact, nonlinear photonic phenomena and devices in silicon, such as Raman amplification and lasing [1]-[4], optical modulation [5], [6], wavelength conversion [7]-[9], all-optical switching [10]-[12], pulse compression [13], [14], control of slow light [15], [16] and so on, have been successfully demonstrated. Two-photon absorption (TPA) and TPA-induced free-carrier absorption (FCA) are nonlinear losses dominating at high intensities. Because they introduce additional loss, so far they have been considered as detrimental effects in nonlinear photonic silicon devices. However, engineered use of TPA and FCA may increase the capabilities of silicon photonics in different applications [13], [17]. Recently, a novel approach to utilize TPA and FCA losses for ultrafast pulse shaping and mode-locked pulse generation has been demonstrated. We experimentally show that without contribution from group velocity dispersion and self-phase-modulation-induced pulse shaping, the TPA and TPA-induced free carriers alone can deliver short mode-locked optical pulses [13]. Here we show that created mode-locked

Manuscript received December 25, 2007; revised April 14, 2008.

$\mathrm{X}$. Sang is with the Department of Electrical Engineering and Computer Science, University of California, Irvine, CA 92697 USA. He is also with Key Laboratory of Optical and Lightwave Technologies (MOE), Beijing University of Posts and Telecommunications, Beijing 100876, China (e-mail: xzsang@gmail. com).

E.-K. Tien, N. S. Yuksek, F. Qian, Q. Song, and O. Boyraz are with the Department of Electrical Engineering and Computer Science, University of California, Irvine, CA 92697 USA (e-mail: oboyraz@uci.edu).

Color versions of one or more of the figures in this letter are available online at http://ieeexplore.ieee.org.

Digital Object Identifier 10.1109/LPT.2008.925493

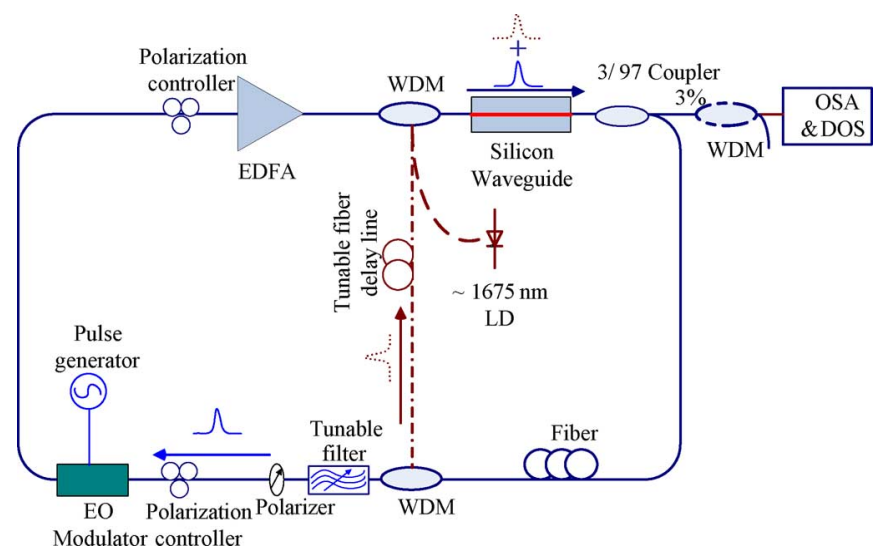

Fig. 1. Schematic of the experimental setup.

pulses can stimulate a Raman effect in the same silicon waveguide and provide dual wavelength lasing. Dual-wavelength mode-locked laser pulses operating at 1540 and $1675 \mathrm{~nm}$ are also experimentally demonstrated. These results indicate that TPA and other nonlinear effects in silicon, such as Raman and four-wave mixing, can be simultaneously utilized to enhance the functionality of the silicon chip without using an external pump laser. In particular, the proposed technique will be available at longer wavelengths where light sources are sparsely available.

\section{PUlse COMPRESSION AND RAMAN AMPLIFICATION}

\section{A. Principle of Pulse Compression}

The principle of laser mode-locking in a silicon waveguide has been previously described in detail [13], [14]. Laser mode-locking relies on transient behavior of free carriers, which are created by TPA. Since free-carrier lifetime is much larger than the optical pulsewidth, the generated free carriers accumulate and introduce time-dependent attenuation along the pulse. As a result, the trailing edge of the pulse entering the silicon waveguide is attenuated by TPA and free carriers that are created by the front edge of the same pulse. This transient behavior of free-carrier concentration will build up over the pulse duration every time the pulse propagates through the silicon waveguide and facilitate self-compression and mode-locking inside a laser cavity. Fig. 1 illustrates the schematic of the experimental setup used for mode-locking. Here, a 1.7-cm-long silicon-on-insulator waveguide with a $\sim 5-\mu \mathrm{m}^{2}$ effective area is used for pulse compression. The waveguide has a $\mathrm{p}-\mathrm{i}-\mathrm{n}$ diode structure to inject carriers and hence has a modulation capability. The modulation feature has not been utilized in this experiment due to its long resistive-capacitive-time constant, which results in the generation of wide output pulses. The 


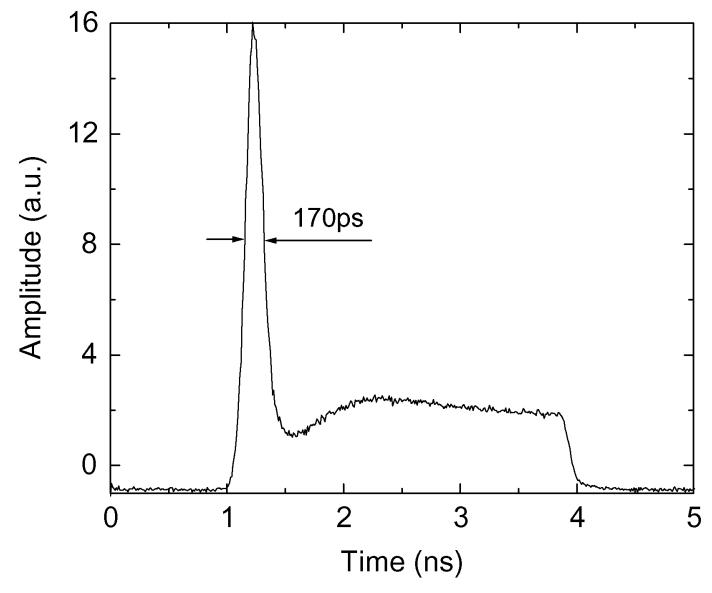

Fig. 2. Compressed pulse by TPA and FCA.

output of the waveguide is connected to a 3/97 tap coupler where the $3 \%$ port is used as an output and the $97 \%$ port is fed into the gain medium, a high power erbium-doped fiber amplifier (EDFA). A resonator is formed by connecting the EDFA output back into the silicon waveguide input end. A tunable filter is inserted to ensure lasing at a desired wavelength and suppress undesired amplified spontaneous emission accumulation. Since the pulse shaping by free carriers requires a time-varying optical signal circulating inside the cavity, an electrooptic (EO) modulator is connected to the waveguide to start initial pulsation. The output pulsewidth is expected to be minimum when the frequency of the function generator matches the fundamental cavity frequency. Here, frequency locking is achieved by simultaneously monitoring the pulse shape and the modulation frequency. The output of the resonator is connected to an optical spectrum analyzer (OSA) and a photodetector followed by a $25-\mathrm{GHz}$ sampling digital oscilloscope (DOS) that measures output pulse characteristics.

\section{B. Raman Amplification Using Compressed Pulses}

Net gain amplification and lasing in silicon have been previously demonstrated using a pulsed-pumping technique [1], [3]. To study stimulated Raman scattering inside the mode-locked laser cavity, a simple pump-probe experiment is conducted by using a continuous-wave (CW) probe laser around $1675 \mathrm{~nm}$. The setup is shown in Fig. 1. In this experiment, Raman gain inside the laser cavity is measured for various pulsing conditions, as illustrated in Fig. 2. We show that a 2.8-dB Raman gain can be measured at the front end of a long pulse with a large pedestal when the mode-locking condition is not optimized, as shown in Fig. 3. However, at the same time, the pulse suffers from a 7-dB free-carrier loss at the trailing edge. Hence, for low loss and an effective Raman effect, the fundamental pulse mode-locking should be optimized before the Raman cavity is formed.

\section{Generation Dual Wavelength Short Pulses}

The observation of a 2.8- $\mathrm{dB}$ gain indicates that a careful design of the laser cavity can facilitate lasing at the Stokes wavelength by stimulated Raman scattering without an additional pump and hence enhance the functionality of the silicon chip. Because of wavelength selectivity of the filter, another cavity for Raman Stokes pulse is needed. To facilitate resonance at the pump wavelength and the Stokes wavelength, the experimental

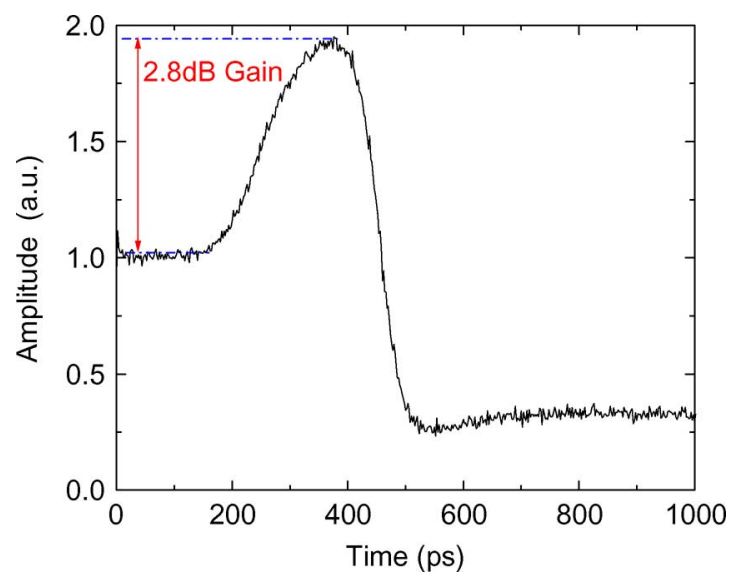

Fig. 3. Raman gain pumped with compressed pulses.

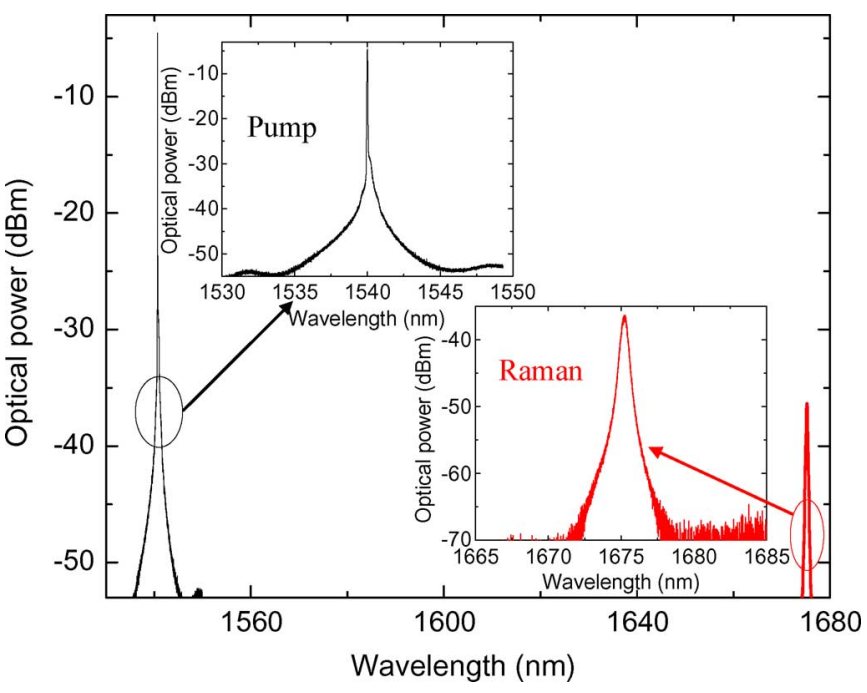

Fig. 4. Spectra of the pulse-compressed pump and Raman laser.

setup is modified according to dashed line in Fig. 1. After removing the probe $\mathrm{CW}$ laser, the original laser cavity is modified by using two wavelength-division-multiplexing couplers, which separate the Stokes from the wavelength sensitive components in the pulse compression cavity and recombine before the silicon waveguide, and a tunable fiber delay. Once mode-locking is optimized to generate short pulses, we are able to utilize the short pulses as the pump to achieve stimulated Raman scattering and mode-locking in the second cavity. After pulses at both wavelengths are aligned temporally, we observe lasing at Stokes and pump wavelengths. The spectra of the dual wavelength laser are shown in Fig. 4. The pump wavelength is selected by the bandpass filter inside the laser cavity to be $1540 \mathrm{~nm}$ and the expected Raman Stokes signal is at wavelength of $1675 \mathrm{~nm}$ [3].

Fig. 5(a) illustrates the 100-ps mode-locked pulses generated inside the main laser cavity. Due to the polarization sensitivity of the mode-locking and Raman processes, we observe that the fundamental pulsewidth is slightly broader than pure mode-locking optimum and it is difficult to simultaneously achieve mode-locking and Raman lasing. Fig. 5(b) shows the Raman Stokes pulse observed at the output. Since free carriers are created and accumulate in the silicon waveguide, the Raman Stokes pulse also suffers from free-carrier attenuation. 


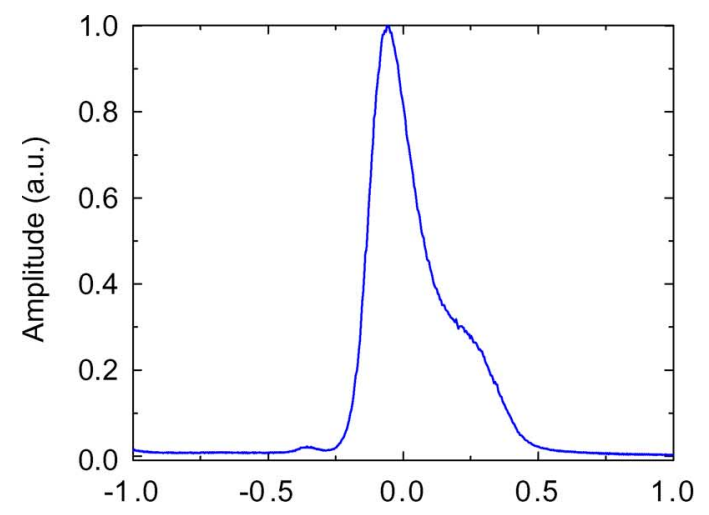

(a)

Time (ns)

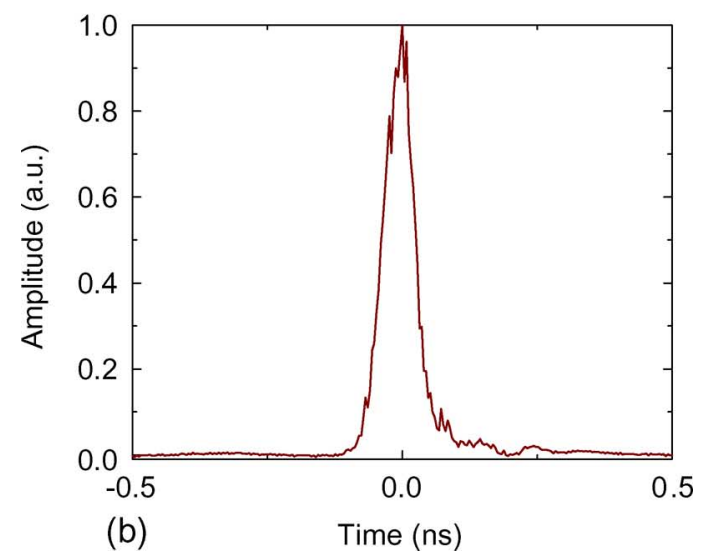

Fig. 5. Time profiles for (a) the compressed pump and (b) Raman pulses.

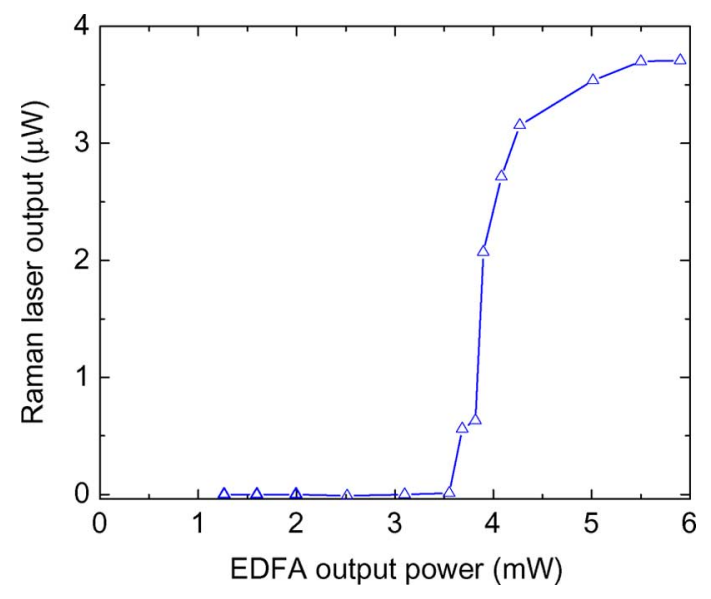

Fig. 6. Raman laser output power as a function of EDFA output power.

Therefore, the final outcome of the Raman Stokes pulses is the combination of Raman amplification, TPA, and free-carrier effect. To measure the lasing threshold at the Stokes wavelength, we change the pump power circulating inside the laser cavity. Fig. 6 illustrates the variation in Stokes power with respect to EDFA output power sweep from 0 to $6 \mathrm{~mW}$. The lasing threshold is measured to be $\sim 3.75 \mathrm{~mW}$. Since the pump pulsewidth is a function of EDFA output power, we observe different Stokes pulsewidths at different EDFA output power levels. Increasing the EDFA output power above $5 \mathrm{~mW}$ causes the Raman output power to enter into saturation because of nonlinear losses including TPA and FCA.

\section{CONCLUSION}

Laser mode-locking is achieved by pulse compression due to TPA and TPA-induced FCA. We also demonstrate that the generated short pulse can also act as a pump for stimulated Raman scattering in the same silicon chip and provide dual wavelength lasing. Laser mode-locking and dual wavelength lasing at 1540 and $1675 \mathrm{~nm}$ are experimentally achieved using a silicon waveguide in the dual-fiber-ring-cavity with an EDFA and silicon as the gain media. The performance of pulse compression in the silicon waveguide can be enhanced using small core waveguides [12]-[14], which can stimulate the Raman effect at even lower powers. Additionally, the broadband nature of this mode-locking scheme may deliver short pulses and facilitate nonlinear optical devices at wavelengths where conventional saturable absorber mode-locking fails.

\section{REFERENCES}

[1] O. Boyraz and B. Jalali, "Demonstration of 11dB fiber-to-fiber gain in a silicon Raman amplifier," Electron. Express, vol. 1, no. 14, pp. 429-434, 2004.

[2] A. Liu, H. Rong, R. Jones, O. Cohen, D. Hak, and M. Paniccia, “Optical amplification and lasing by stimulated Raman scattering in silicon waveguides," J. Lightw. Technol., vol. 24, no. 3, pp. 1440-1455, Mar. 2006.

[3] O. Boyraz and B. Jalali, "Demonstration of a silicon Raman laser," Opt. Express, vol. 12, no. 21, pp. 5269-5273, 2004.

[4] H. Rong et al., "A continuous-wave Raman silicon laser," Nature, vol. 433, no. 7027, pp. 725-728, Feb. 17, 2005.

[5] A. Liu et al., "A high-speed silicon optical modulator based on a metal-oxide-semiconductor capacitor," Nature, vol. 427, no. 6975, pp. 615-618, Feb. 12, 2004.

[6] A. Liu, L. L. Liao, D. Rubin, H. Nguyen, B. Ciftcioglu, Y. Chetrit, N. Izhaky, and M. Paniccia, "High-speed optical modulation based on carrier depletion in a silicon waveguide," Opt. Express, vol. 15, pp. 660-668, 2007.

[7] V. Raghunathan, R. Claps, D. Dimitropoulos, and B. Jalali, "Parametric Raman wavelength conversion in scaled silicon waveguides," J. Lightw. Technol., vol. 23, no. 6, pp. 2094-2102, Jun. 2005.

[8] K. Yamada, H. Fukuda, T. Tsuchizawa, T. Watanabe, T. Shoji, and S. Itabashi, "All-optical efficient wavelength conversion using silicon photonic wire waveguide," IEEE Photon. Technol. Lett., vol. 18, no. 9, pp. 1046-1048, May 1, 2006.

[9] K. K. Tsia, S. Fathpour, and B. Jalali, "Energy harvesting in silicon wavelength converters," Opt. Express, vol. 14, no. 25, pp. 12327-12333, 2006.

[10] O. Boyraz, P. Koonath, V. Raghunathan, and B. Jalali, "All optical switching and continuum generation in silicon waveguides," Opt. Express, vol. 12, no. 17, pp. 4094-4102, 2004.

[11] V. R. Almeida et al., "All-optical control of light on a silicon chip," Nature, vol. 431, no. 7012, pp. 1081-1084, Oct. 28, 2004.

[12] V. R. Almeida, C. A. Barrios, and R. R. Panepucci et al., "All-optical switching on a silicon chip," Opt. Lett., vol. 29, no. 24, pp. 2867-2869, Dec. 2005.

[13] E. Tien, N. S. Yuksek, F. Qian, and O. Boyraz, "Pulse compression and modelocking by using TPA in silicon waveguides," Opt. Express, vol. 15, pp. 6500-6506, May 2007.

[14] E. Tien, F. Qian, N. S. Yuksek, and O. Boyraz, "Influence of nonlinear loss competition on pulse compression and nonlinear optics in silicon," Appl. Phys. Lett., vol. 91, pp. 201115-1-201115-3, Nov. 2007.

[15] Y. Okawachi et al., "All-optical slow-light on a photonic chip," Opt. Express, vol. 14, no. 6, pp. 2317-2322, Mar. 2006.

[16] F. Xia, L. Sekaric, and Y. Vlasov, "Ultracompact optical buffers on a silicon chip," Nature Photon., vol. 1, no. 1, pp. 65-71, Jan. 2007.

[17] T. K. Liang, L. R. Nunes, and T. Sakamoto et al., "Ultrafast all-optical switching by cross-absorption modulation in silicon wire waveguides," Opt. Express, vol. 13, no. 19, pp. 7298-7303, 2005. 